\title{
Neuropharmacological Dissection of Placebo Analgesia: Expectation-Activated Opioid Systems versus Conditioning- Activated Specific Subsystems
}

\author{
Martina Amanzio and Fabrizio Benedetti \\ Department of Neuroscience and Centro Interuniversitario per la Neurofisio logia del Dolore Center for the \\ Neurophysiology of Pain, University of Torino Medical School, 10125 Torino, Italy
}

We investigated the mechanisms underlying the activation of endogenous opioids in placebo analgesia by using the model of human experimental ischemic arm pain. Different types of placebo analgesic responses were evoked by means of cognitive expectation cues, drug conditioning, or a combination of both. Drug conditioning was performed by means of either the opioid agonist morphine hydrochloride or the nonopioid ketorolac tromethamine. Expectation cues produced placebo responses that were completely blocked by the opioid antagonist naloxone. Expectation cues together with morphine conditioning produced placebo responses that were completely antagonized by naloxone. Morphine conditioning alone (without expectation cues) induced a naloxone-reversible placebo effect. By contrast, ketorolac conditioning together with expectation cues elicited a placebo effect that was blocked by naloxone only partially. Ketorolac conditioning alone produced placebo

The neurobiology of placebo was born when Levine et al. (1978) discovered that the opioid antagonist naloxone inhibits the placebo analgesic response. There are now several lines of evidence indicating that placebos activate endogenous opioid systems, thus producing placebo analgesia (Grevert et al., 1983; Fields and Levine, 1984; Levine and Gordon, 1984; Benedetti et al., 1995; Benedetti, 1996; Benedetti and Amanzio, 1997; Fields and Price, 1997). However, Gracely et al. (1983) showed that placebo analgesia may also occur without the involvement of endogenous opioid systems. In addition, in the study by Grevert et al. (1983) naloxone blocked placebo analgesia only partially, suggesting that both opioid and nonopioid components play an important role.

The activation of opioid or nonopioid systems represent only the final pathway of a complex mechanism that is poorly understood. In the typical paradigm used to produce placebo analgesia, a substance known to be nonanalgesic (e.g., saline solution) is administered, and the subject is told that it is a powerful painkiller. At least two theories have been proposed to explain this phenomenon as the basis for the activation of endogenous opioids. First, cognitive factors, like expectation of pain relief, are supposed to trigger the release of opioids in the CNS (for review,

Received Aug. 28, 1998; revised Oct. 14, 1998; accepted Oct. 21, 1998.

We thank the two reviewers for their important criticisms. They helped us to improve the article. This work was supported by grants from Ministero dell’Universitá e della Ricerca Scientifica e Tecnologica and Consiglio Nazionale delle Ricerche "Coordinate Project on Trigeminal Pain".

Correspondence should be addressed to Fabrizio Benedetti, Dipartimento di Neuroscienze, Università di Torino, Corso Raffaello 30, 10125 Torino, Italy. Copyright (C) 1998 Society for Neuroscience $\quad 0270-6474 / 98 / 190484-11 \$ 05.00 / 0$ responses that were naloxone-insensitive. Therefore, we evoked different types of placebo responses that were either naloxone-reversible or partially naloxone-reversible or, otherwise, naloxone-insensitive, depending on the procedure used to evoke the placebo response. These findings show that cognitive factors and conditioning are balanced in different ways in placebo analgesia, and this balance is crucial for the activation of opioid or nonopioid systems. Expectation triggers endogenous opioids, whereas conditioning activates specific subsystems. In fact, if conditioning is performed with opioids, placebo analgesia is mediated via opioid receptors, if conditioning is performed with nonopioid drugs, other nonopioid mechanisms result to be involved.

Key words: pain; placebo analgesia; cognition; conditioning; morphine; nonsteroid anti-inflammatory drugs; endogenous opioids

see Benedetti and Amanzio, 1997; Fields and Price, 1997). Second, a classical conditioning mechanism has been proposed, in which repeated associations between active analgesics, pain relief, and therapeutic surroundings produce a conditioned placebo analgesic response (Wickramasekera, 1985; Voudouris et al., 1989, 1990; Benedetti and Amanzio, 1997; Fields and Price, 1997; Price and Fields, 1997). In addition, the anxiety theory postulates that placebo analgesia is caused by a reduction of anxiety (Evans, 1985), whereas the response-appropriate sensation theory proposes that the global experience of pain results from a complex internal analysis of different brain states (Wall, 1993). These theories are not necessarily in conflict because each of them may represent a different aspect of the same phenomenon (Wall, 1992).

Therefore, although there is now a general agreement on the involvement of endogenous opioids in some types of placebo analgesia (ter Riet et al., 1998), the mechanisms of their activation is not known. As stressed by Fields and Levine (1984), it is necessary to understand the conditions and the mechanisms capable to produce naloxone-reversible and naloxone-insensitive placebo responses. On the basis of Fields and Levine's considerations, we analyzed different types of placebo analgesia that were induced by different combinations of expectation cues and conditioning procedures, and by different opioid and nonopioid conditioning drugs. In such a way, we could perform a true neuropharmacological dissection of placebo analgesia into opioid and nonopioid components and could identify how these neurochemical systems are related to cognitive and conditioning mechanisms. 


\begin{tabular}{|c|c|c|c|c|}
\hline Group & $\begin{array}{l}\text { Number } \\
\text { of subjects }\end{array}$ & $\begin{array}{l}\text { Sex } \\
\text { (male/female) }\end{array}$ & Age (years) & Weight $(\mathrm{kg})$ \\
\hline 1 & 56 & $31 / 25$ & $47.3 \pm 7.6$ & $61.2 \pm 10.5$ \\
\hline 2 & 25 & $15 / 10$ & $49.9 \pm 8.3$ & $63.5 \pm 12.4$ \\
\hline 3 & 16 & $9 / 7$ & $45.1 \pm 9.9$ & $58.2 \pm 9.7$ \\
\hline 4 & 15 & $9 / 6$ & $50.5 \pm 8.7$ & $57.6 \pm 9.3$ \\
\hline 5 & 13 & $8 / 5$ & $51.1 \pm 11.4$ & $59.8 \pm 10.4$ \\
\hline 6 & 14 & $7 / 7$ & $47.7 \pm 9.2$ & $60.6 \pm 10.8$ \\
\hline 7 & 14 & $8 / 6$ & $46.3 \pm 11.8$ & $60.1 \pm 8.5$ \\
\hline 8 & 16 & $10 / 6$ & $47.5 \pm 7.9$ & $60.9 \pm 9.0$ \\
\hline 9 & 17 & $10 / 7$ & $48.2 \pm 9.5$ & $58.8 \pm 9.7$ \\
\hline 10 & 15 & $9 / 6$ & $50.0 \pm 8.8$ & $59.5 \pm 10.6$ \\
\hline 11 & 14 & $9 / 5$ & $45.9 \pm 10.0$ & $62.4 \pm 11.1$ \\
\hline 12 & 14 & $7 / 7$ & $49.2 \pm 7.8$ & $61.0 \pm 8.9$ \\
\hline
\end{tabular}

Part of this study has been published in abstract form (Amanzio et al., 1998).

\section{MATERIALS AND METHODS}

Subjects. A total of 229 subjects participated in the study after they signed a written informed consent in which the experimental procedure was described, and the use of morphine, ketorolac, and naloxone was explained in detail. In particular, they were told that these drugs were not dangerous and did not produce side effects at the doses used in the study. Each subject underwent a clinical examination in which blood pressure and electrocardiogram were recorded. All subjects with heart problems were not allowed to participate in the study. Most of the subjects referred a previous experience with analgesics, either opioids or nonopioids, for different types of pathological conditions (e.g., headache or previous surgery). All the experimental procedures were conducted in conformance with the policies and principles contained in the Declaration of Helsinki. The 229 subjects were subdivided into 12 groups, whose characteristics are shown in Table 1. It should be noted that the ratio of males to females, age, and weight did not differ among the different groups.

Pain stimulus. Pain was induced experimentally by means of the tourniquet technique. This test produces ischemic pain of the arm that increases over time (Smith et al., 1966, 1972; Benedetti, 1996). To avoid variability among different subjects, we induced a quick increase of pain according to the following procedure. The subject reclined on a bed, his or her nondominant forearm was extended vertically, and venous blood was drained by means of an Esmarch bandage. A sphygmomanometer was placed around the upper arm and inflated to a pressure of 300 $\mathrm{mmHg}$. The Esmarch bandage was maintained around the forearm, which was lowered on the subject's side. After this, the subject started squeezing a hand spring exerciser 12 times while his or her arm rested on the bed. Each squeeze was timed to last $2 \mathrm{sec}$, followed by a $2 \mathrm{sec}$ rest. The force necessary to bring the handles together was $7.2 \mathrm{~kg}$. This type of ischemic pain increases over time very quickly, and the pain becomes unbearable after about 13-14 min (Table 2). A timer was started after the last squeeze, and the subject stopped the timer when the pain became unbearable. At this point, the experiment was discontinued, and the time was recorded. Thus, pain tolerance was defined as the time from the last squeeze to unbearable pain.

Drug administration. All drugs used in the present study were administered through an intravenous line. Before starting the experimental procedure, a needle was inserted into a vein of the dominant forearm. The needle was connected to a line, $1 \mathrm{~m}$ long, through which a slow infusion of 5\% glucose solution was administered. The intravenous line reached a screen behind the subject's bed. In such a way, hidden injections could be performed by the experimenter. Naloxone (Crinos, Italy) was administered at a dose of $0.14 \mathrm{mg} / \mathrm{kg}$ in sterile solution of $\mathrm{NaCl}$ $0.9 \%$. The infusion rate (controlled by an infusion pump) was $0.1 \mathrm{ml} / \mathrm{sec}$ for a total infusion time ranging from 180 to $250 \mathrm{sec}$. The conditioning drugs were morphine hydrochloride and ketorolac tromethamine. Morphine hydrochloride is an opioid agonist and was administered at a dose of $0.12 \mathrm{mg} / \mathrm{kg}$ in sterile solution of $\mathrm{NaCl} 0.9 \%$, with an infusion rate of $0.1 \mathrm{ml} / \mathrm{sec}$ (total infusion time ranging from 70 to $100 \mathrm{sec}$ ). Ketorolac tromethamine (Formit, Italy) is a nonsteroid anti-inflammatory drug (NSAID) with no activity on opioid receptors, and was administered at a dose of $0.43 \mathrm{mg} / \mathrm{kg}$ in sterile solution of $\mathrm{NaCl} 0.9 \%$, with an infusion rate of $0.1 \mathrm{ml} / \mathrm{sec}$ (total infusion time ranging from 70 to $110 \mathrm{sec}$ ).

Experimental procedure. The experiments were performed according to a randomized double-blind design in which neither the subject nor the experimenter knew what drug was administered. To do this, either morphine or saline were given on days 2 and 3. Similarly, either ketorolac or saline were given on days 2 and 3 . On day 4, either morphine or naloxone or saline were administered. To avoid a large number of subjects, only two or three subjects per group received saline on days 2 and 3 and morphine or ketorolac on day 4 . These subjects were not included in the study because they were used only to allow the doubleblind design. By using this experimental approach, we were completely blind to morphine, ketorolac, and naloxone. All drugs were administered $10 \mathrm{~min}$ before inflating the sphygmomanometer cuff, and the time interval from cuff inflation to the last squeeze was $1 \mathrm{~min}$. Thus, the time interval from drug administration to last squeeze was the same in all subjects $(11 \mathrm{~min})$. The complete experimental procedure is shown in Figure 1. Group 1 (natural history) was tested with the tourniquet technique for 4 consecutive days without receiving any treatment. Group 2 received a hidden injection of naloxone performed through the intravenous line behind the screen on days 2 and 4 , to ascertain whether naloxone per se affected the ischemic pain. It is important to emphasize that this group did not know that any injection was performed. Group 3 received an open injection (in full view of the subject) of saline $(\mathrm{NaCl}$ $0.9 \%$ solution) on day 2 and was told that it was a powerful painkiller (placebo with expectation of pain relief). Group 4 received an open injection of naloxone on day 2 and was told that it was a potent painkiller (placebo with expectation plus naloxone). Group 5 was treated with

Table 2. The left columns show the pain tolerance baseline on day 1 in all experimental groups and the comparisons with the natural history group on day 1 ( $p$ levels). The right columns show the pain tolerance on the day after drug administration and its comparison with pain tolerance on day 1

\begin{tabular}{|c|c|c|c|c|}
\hline Group & $\begin{array}{l}\text { Pain tolerance base- } \\
\text { line on day } 1(\mathrm{~min})\end{array}$ & $\begin{array}{l}\text { Comparison with natural } \\
\text { history on day } 1 \text { (ANOVA) }\end{array}$ & $\begin{array}{l}\text { Pain tolerance on the day } \\
\text { after experimental test (min) }\end{array}$ & $\begin{array}{l}\text { Comparison with day } 1 \\
\text { (ANOVA) }\end{array}$ \\
\hline 2 & $13.32 \pm 4.2$ & $F_{(1,79)}=0.26 ; p=0.612$ & (day 3) $13.24 \pm 3.03$ & $F_{(1,24)}=0.01 ; p=0.910$ \\
\hline 3 & $14.12 \pm 4.38$ & $F_{(1,70)}=0.04 ; p=0.838$ & (day 3) $13.87 \pm 3.83$ & $F_{(1,15)}=0.33 ; p=0.572$ \\
\hline 4 & $13.33 \pm 4.25$ & $F_{(1,69)}=0.17 ; p=0.683$ & (day 3) $12.53 \pm 3.64$ & $F_{(1,14)}=2.09 ; p=0.171$ \\
\hline 5 & $12.77 \pm 4.13$ & $F_{(1,67)}=0.64 ; p=0.427$ & (day 5) $12.92 \pm 2.9$ & $F_{(1,12)}=0.03 ; p=0.862$ \\
\hline 6 & $12.14 \pm 4.49$ & $F_{(1,68)}=1.64 ; p=0.204$ & (day 5) $11.71 \pm 4.43$ & $F_{(1,13)}=0.40 ; p=0.538$ \\
\hline 7 & $13.57 \pm 3.72$ & $F_{(1,68)}=0.05 ; p=0.824$ & (day 5) $13.43 \pm 3.11$ & $F_{(1,13)}=0.04 ; p=0.842$ \\
\hline 8 & $13.69 \pm 4.27$ & $F_{(1,70)}=0.02 ; p=0.893$ & (day 5) $13.19 \pm 4.02$ & $F_{(1,15)}=0.48 ; p=0.497$ \\
\hline 9 & $14.35 \pm 4.14$ & $F_{(1,71)}=0.16 ; p=0.690$ & (day 5) $13.71 \pm 3.33$ & $F_{(1,16)}=1.39 ; p=0.256$ \\
\hline 10 & $13.27 \pm 3.77$ & $F_{(1,69)}=0.22 ; p=0.643$ & (day 5) $12.67 \pm 3.29$ & $F_{(1,14)}=0.93 ; p=0.352$ \\
\hline 11 & $13.86 \pm 4.07$ & $F_{(1,68)}=0.00 ; p=0.999$ & (day 5) $13.21 \pm 3.72$ & $F_{(1,13)}=0.84 ; p=0.375$ \\
\hline 12 & $12.86 \pm 4.24$ & $F_{(1,68)}=0.57 ; p=0.454$ & (day 5) $12.29 \pm 3.22$ & $F_{(1,13)}=0.55 ; p=0.470$ \\
\hline
\end{tabular}




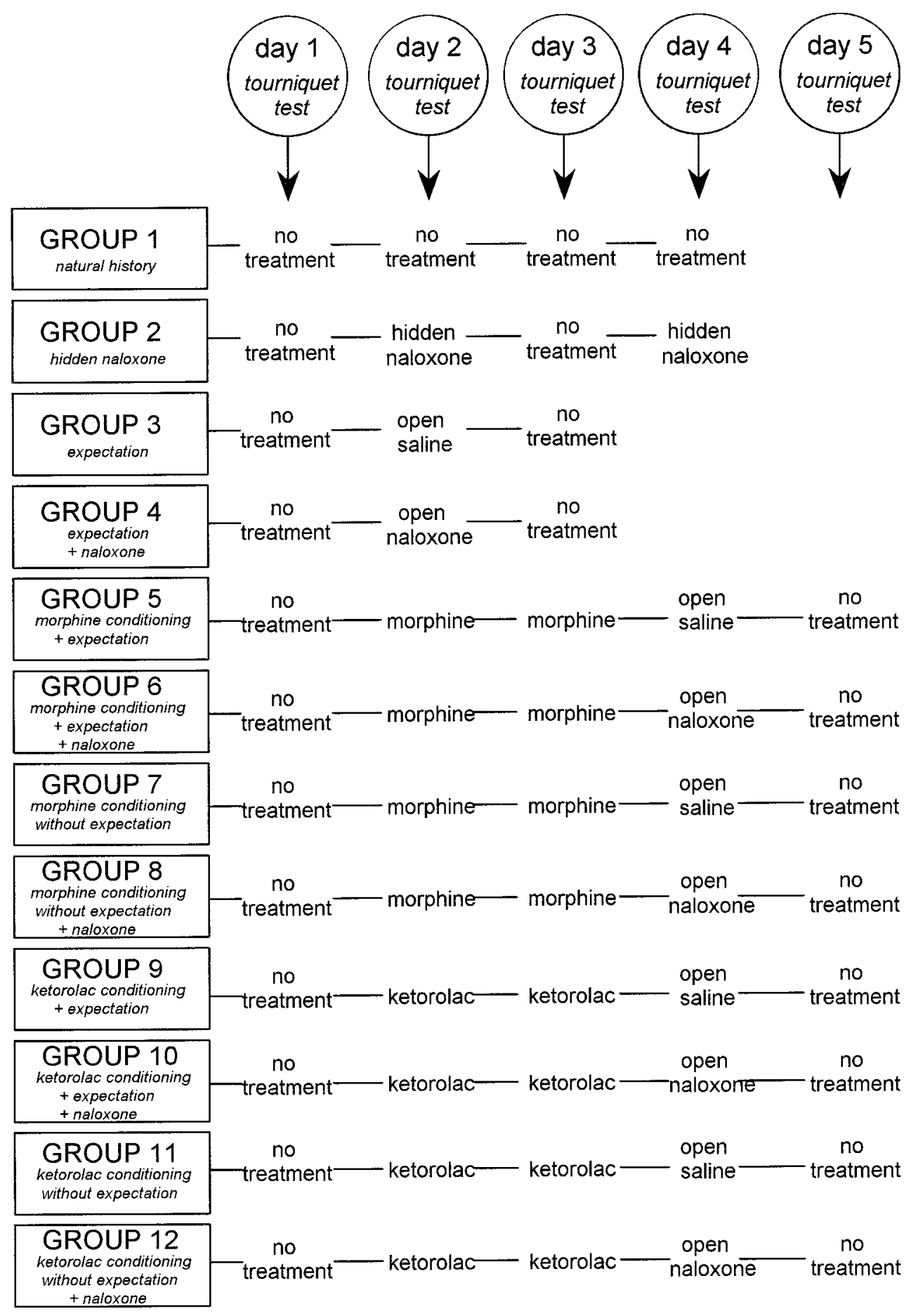

Figure 1. Experimental paradigm used in the study to identify the opioid and nonopioid components of placebo analgesia. Below each group the experimental condition is specified. No treatment means that the tourniquet test was performed without infusion of any drug.

morphine (open injection) on days 2 and 3 (conditioning) and received an open injection of saline on day 4, believing that it was morphine (placebo with expectation). Group 6 was treated with morphine (open injection) on days 2 and 3 (conditioning) and received an open injection of naloxone on day 4 , believing that it was morphine (placebo with expectation plus naloxone). Groups 7 and 8 received the same treatment of groups 5 and 6 . However, the open injections of saline or naloxone on day 4 were believed to be a neutral nonanalgesic solution (antibiotic) used for sterility purposes; in this case, subjects did not expect any pain relief (placebo without expectation but with previous conditioning).
Groups 9-12 were treated as groups 5-8, with the exception that conditioning on days 2 and 3 was performed with the nonopioid ketorolac.

The verbal instructions used in the different experimental conditions are reported below. In the conditioning procedures with either morphine or ketorolac on days 2 and 3, subjects were told that the drugs were potent analgesics producing a quick pain reduction and, therefore, an increase of tolerance. On day 4, in the expectation procedure (groups 5, 6, 9, and 10), subjects were told that the drug was the same potent analgesic used on days 2 and 3. By contrast, in the no-expectation procedure (groups 7, 8, 11 , and 12), subjects were told that the drug was an antibiotic used "to 
A

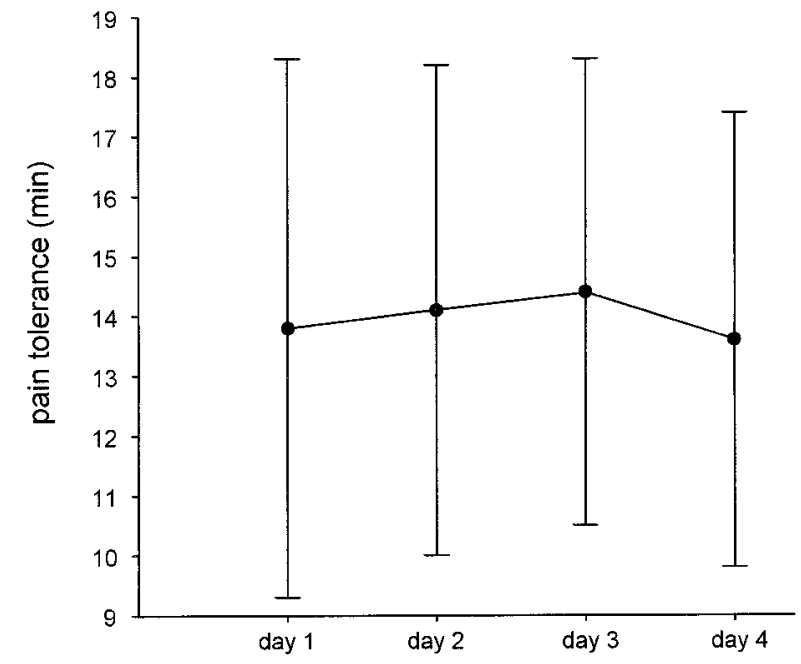

B

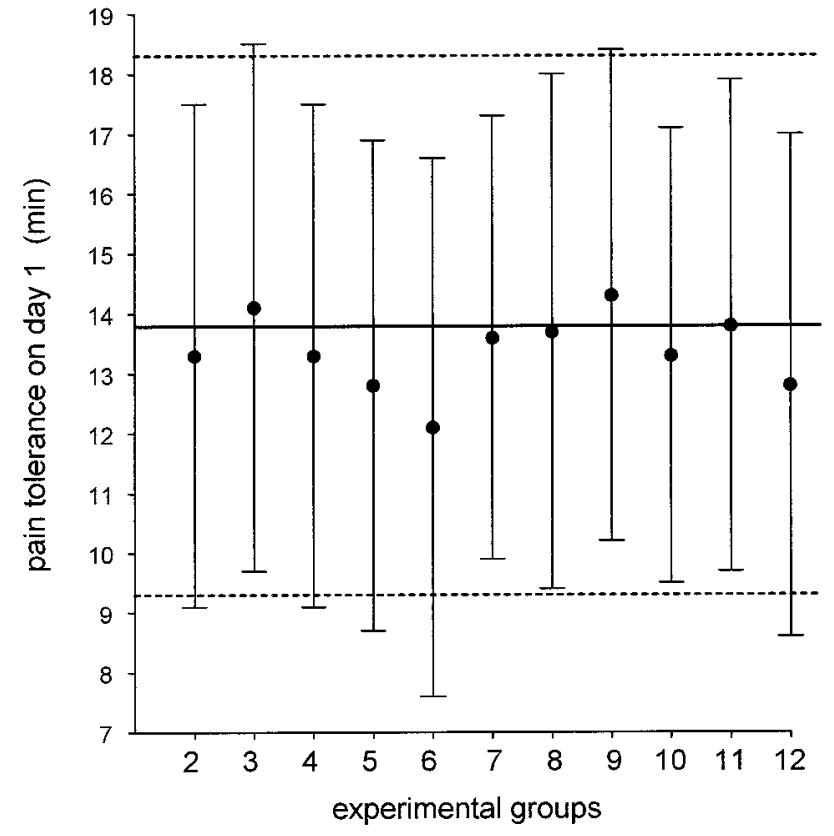

Figure 2. Analysis of the natural history of ischemic pain. $A$, Means and SDs of the natural history are shown for group 1 on 4 consecutive days. $B$, Pain baseline (mean \pm SD) on day 1 in all groups and comparison with the natural history group on day 1 . The horizontal bold line represents the mean of day 1 shown in $A$, the broken lines represent the SD. The statistical analysis of the natural history is shown in Table 2.

clean the blood" for the sake of sterility; thus, these subjects believed that day 4 was not used for analgesic tests.

It should be noted that the tourniquet test was performed without any treatment on the first and last days in all groups, and it was used as a control.

Statistical analysis. The differences between and within treatments were tested by means of the ANOVA followed by the Newman-Keuls multiple range test for multiple comparisons. In addition, linear regression analysis was performed by considering the data from single subjects. Therefore, data are presented as mean \pm SD or for single subjects. Differences were considered to be statistically significant at $p<0.05$.

\section{RESULTS}

\section{The natural history of ischemic arm pain}

The natural history group showed no variation of pain tolerance when the tourniquet test was repeated for 4 consecutive days
$\left(F_{(3,165)}=1.5 ; p=0.216\right)$, indicating that the tourniquet technique produces pain tolerances that remain constant for several days (Fig. $2 A$ ). In all groups, the pain tolerance baseline on day 1 did not differ from the mean value of the natural history group (Fig. 2B). It can also be seen in Table 2 that no significant difference was found between each group and the natural history on day 1 . In addition, the post-treatment control test (either day 3 for groups 2-4 or day 5 for the other groups) did not differ from the pretreatment control test of day 1 (Table 2). In conclusion, when the tourniquet test was performed without any treatment (controls), it always produced constant and consistent results in a range of time of at least $5 \mathrm{~d}$. Therefore, any departure from this pain tolerance baseline (natural history) can be viewed as a true placebo effect.

\section{Opioid-mediated placebo analgesia}

Before starting the conditioning procedures, we wanted to test whether a placebo effect and its reversal by naloxone could be adequately observed in these experimental conditions. First of all, we tested whether naloxone per se affects this type of experimental pain. A hidden injection of naloxone (group 2) on days 2 and 4 did not produce any variation of pain tolerance compared with days 1 and $3\left(F_{(3,72)}=0.01 ; p=0.991\right)$ (Fig. $\left.3 A\right)$. Then we evoked a placebo response by injecting saline which the subjects believed to be a potent painkiller (group 3); a clearcut placebo effect could be observed compared with days 1 and $3\left(F_{(1,15)}=12.36\right.$; $p<$ 0.003 ) (Fig. $3 B$ ). If, however, the open injection contained naloxone (group 4), no effect could be observed (Fig. $3 C$ ); in fact, no difference was found between days 2 and $1\left(F_{(1,14)}=4.41 ; p=\right.$ $0.054)$. It is worth noting that, albeit nonsignificant, there was a tendency for pain tolerance on day 2 to be smaller than on day 1 . Therefore, we can conclude that this type of experimental pain (1) is not affected by naloxone, (2) can produce placebo responses, and (3) these placebo responses are blocked by naloxone.

\section{Conditioning with morphine hydrochloride}

When morphine was administered on days 2 and 3, a significant increase in pain tolerance was found $\left(F_{(1,12)}=274.46 ; p<0.0001\right.$ and $F_{(1,12)}=157.25 ; p<0.0001$, respectively) (Fig. $4 A$ ). A saline injection on day 4 , which the subjects believed to be morphine (group 5), mimicked the morphine responses of the previous days $\left(F_{(1,12)}=69.12 ; p<0.001\right)$, whereas pain tolerance returned to baseline on day $5\left(F_{(1,12)}=0.03 ; p=0.862\right)($ Fig. $4 A)$. If the same procedure was performed but naloxone, which was believed to be morphine, was injected on day 4 (group 6), no morphinemimicking response could be observed $\left(F_{(1,13)}=0.09 ; p=0.765\right)$ (Fig. $4 B$ ). The same procedure was also used in groups 7 (Fig. $4 C$ ) and 8 (Fig. $4 D$ ). However, the subjects were told that the injection of day 4 was an antibiotic and, thus, they did not expect any pain relief. In group 7 (Fig. 4C), a morphine-mimicking response was found after saline injection on day 4 , even if no expectation of pain relief was present $\left(F_{(1,13)}=78 ; p<0.001\right)$, indicating that the previous morphine conditioning per se was sufficient to evoke a placebo effect. There was a significant difference between the placebo effects of groups 5 (Fig. $4 A$ ) and 7 (Fig. $4 C)\left(F_{(1,25)}=5.5\right.$; $p<0.03)$, indicating that conditioning plus expectation produces a placebo response that is larger than conditioning alone. The conditioning-induced placebo effect was completely blocked by naloxone (group 8) because no effect was observed after an open injection of naloxone that the subjects believed to be an antibiotic $\left(F_{(1,15)}=0.32 ; p=0.580\right)$ (Fig. $\left.4 D\right)$. It is important to note that pain tolerance returned to baseline on day 5 in all cases. 

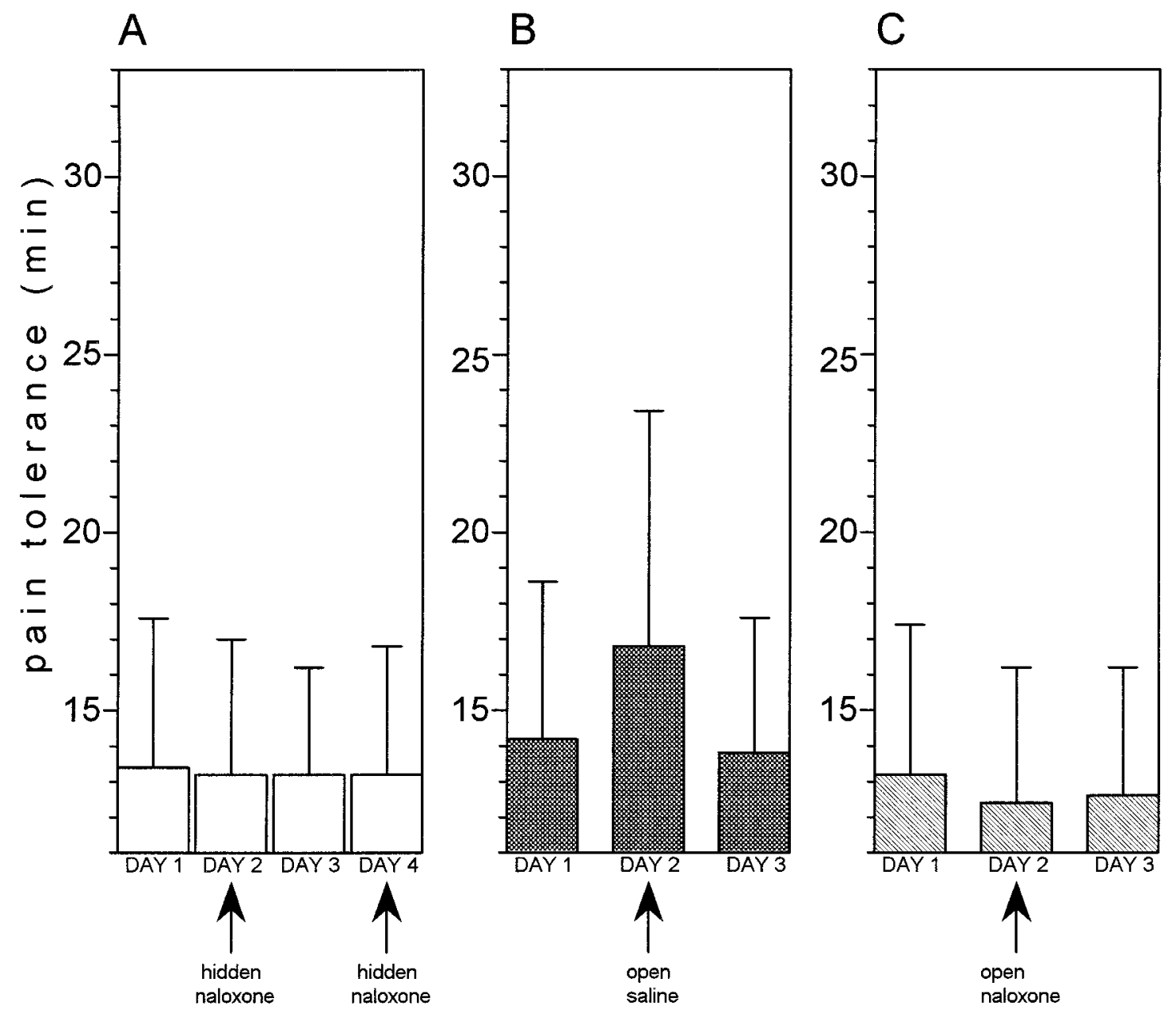

Figure 3. Expectation-induced placebo analgesia and its blockade by naloxone. $A$, A hidden injection of naloxone on days 2 and 4 (group 2 ) does not produce any change in pain tolerance compared with days 1 and 3, indicating that naloxone per se does not affect this type of experimental pain. $B$, An open injection of saline (group 3) produces a placebo analgesic effect. Days 1 and 3 represent preinjection and postinjection controls. $C$, An open injection of naloxone on day 2 (group 4) blocks the placebo effect completely. In fact, pain tolerance on day 2 is equal to preinjection and postinjection controls.

We also performed a linear regression analysis by considering the data from single subjects. We found a high correlation between the response to morphine on day 3 and the response to saline on day 4, according to the rule "the larger the morphine responses, the larger the placebo responses." The analgesic response to morphine was expressed as $\Delta t$, that is, the difference between pain tolerance on days 3 and 1 . Similarly, the analgesic response to placebo was expressed as the difference of pain tolerance on days 4 and 1 . This was true for both groups 5 and 7 $\left(r=0.627 ; t_{(11)}=2.669 ; p<0.025\right.$ and $r=0.855 ; t_{(12)}=5.704$; $p<0.001$, respectively) (Fig. 5, black circles). Naloxone disrupted completely this correlation in both groups 6 and $8\left(r=0.187 ; t_{(12)}\right.$ $=0.661 ; p=0.521$ and $r=-0.282 ; t_{(14)}=-1.1 ; p=0.290$, respectively) (Fig. 5, white circles).

In conclusion, the placebo responses induced by morphine conditioning plus expectation and morphine conditioning alone could be blocked completely by naloxone.

\section{Conditioning with ketorolac tromethamine}

The same procedures used for morphine conditioning and described above were repeated with the nonopioid ketorolac. Administration of ketorolac on days 2 and 3 produced strong analgesic responses $\left(F_{(1,16)}=193.88 ; p<0.0001\right.$ and $F_{(1,16)}=83.22$; $p<0.001$, respectively) (Fig. $6 A$ ). In both groups 9 (Fig. $6 A$ ) and 11 (Fig. $6 C$ ), the saline injection produced ketorolac-mimicking responses $\left(F_{(1,16)}=68.36 ; p<0.001\right.$ and $F_{(1,13)}=28.04 ; p<$ 0.001 , respectively). If naloxone was administered on day 4 (group 10) and was believed to be ketorolac (Fig. 6B), the ketorolacmimicking response was still present $\left(F_{(1,14)}=56 ; p<0.001\right)$, but was significantly smaller than the mimicking response of group 9 $\left(F_{(1,30)}=5.65 ; p<0.025\right)$. Therefore, in this case the placebo response was only partially abolished by naloxone. By contrast, if naloxone was administered on day 4 (group 12) and was believed to be an antibiotic (no-expectation, Fig. 6D), it was completely ineffective in blocking the conditioning-induced placebo response. In fact, the ketorolac-mimicking response was still present $\left(F_{(1,13)}=59.47 ; p<0.001\right)$.

The linear regression analysis performed with the data from single subjects gave the same results (Fig. 7). A correlation between ketorolac responses on day 3 and placebo responses on day 4 was present in groups 9 and 11, which received saline $(r=$ $0.815 ; t_{(15)}=5.44 ; p<0.001$ and $r=0.645 ; t_{(12)}=2.924 ; p<$ 0.015 , respectively) and in groups 10 and 12 , which received naloxone $\left(r=0.580 ; t_{(13)}=2.566 ; p<0.025\right.$ and $r=0.554 ; t_{(12)}$ $=2.308 ; p<0.04$, respectively). 

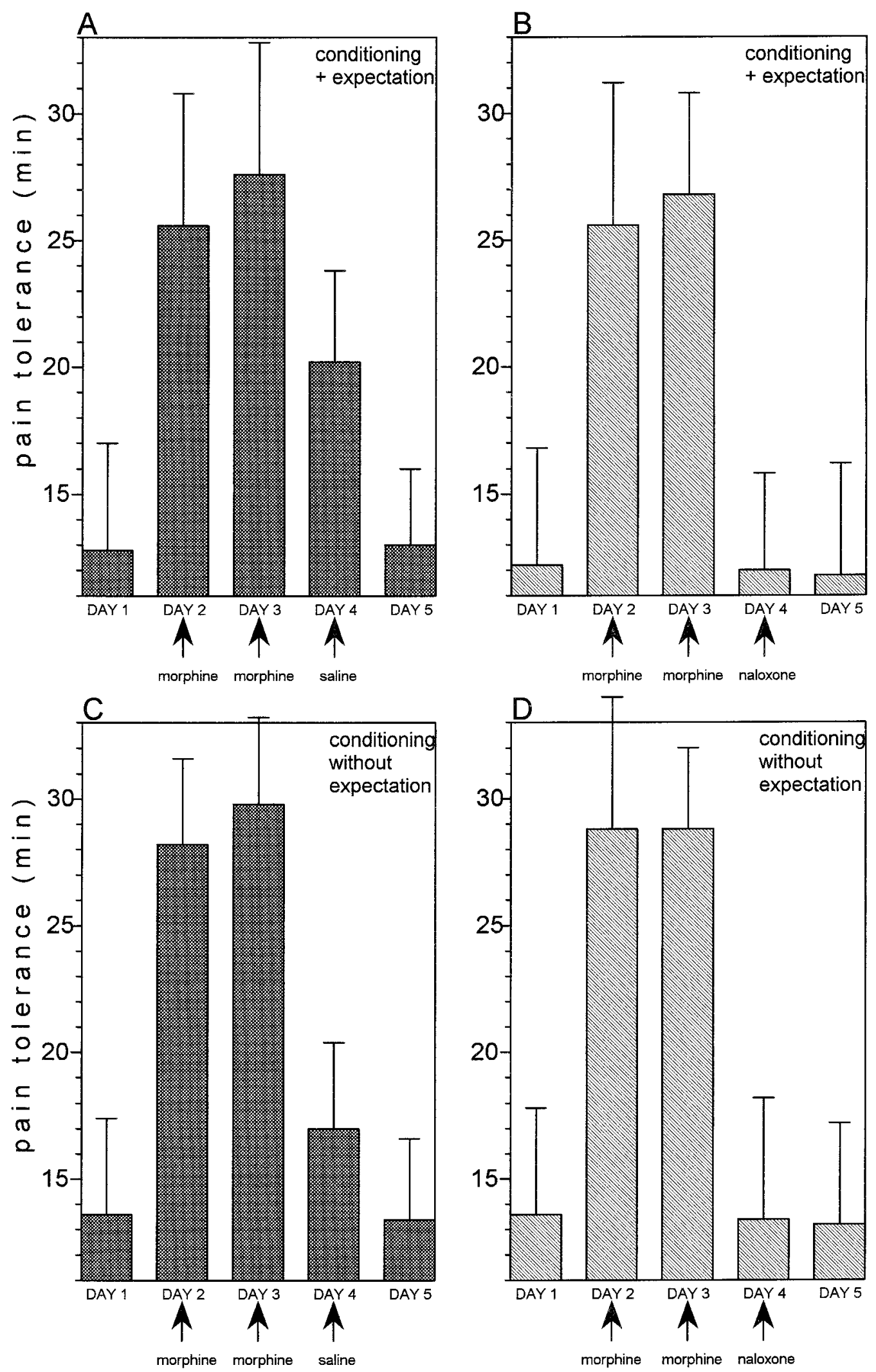

Figure 4. A, After morphine conditioning on days 2 and 3, an open injection of saline, which is believed to be morphine (group 5), mimicks the morphine analgesic response. $B$, If an open injection of naloxone, which is believed to be morphine (group 6), is performed after 2 days of morphine conditioning, the morphine-mimicking effect is completely abolished. $C$, After morphine conditioning for 2 consecutive days, an open injection of saline, which is believed to be an antibiotic (group 7), mimicks the morphine response (albeit less than in $A$ ). $D$, An open injection of naloxone, which is believed to be an antibiotic (group 8), completely blocks the morphine-mimicking effect. In all cases, days 1 and 5 represent preconditioning and postconditioning controls. 

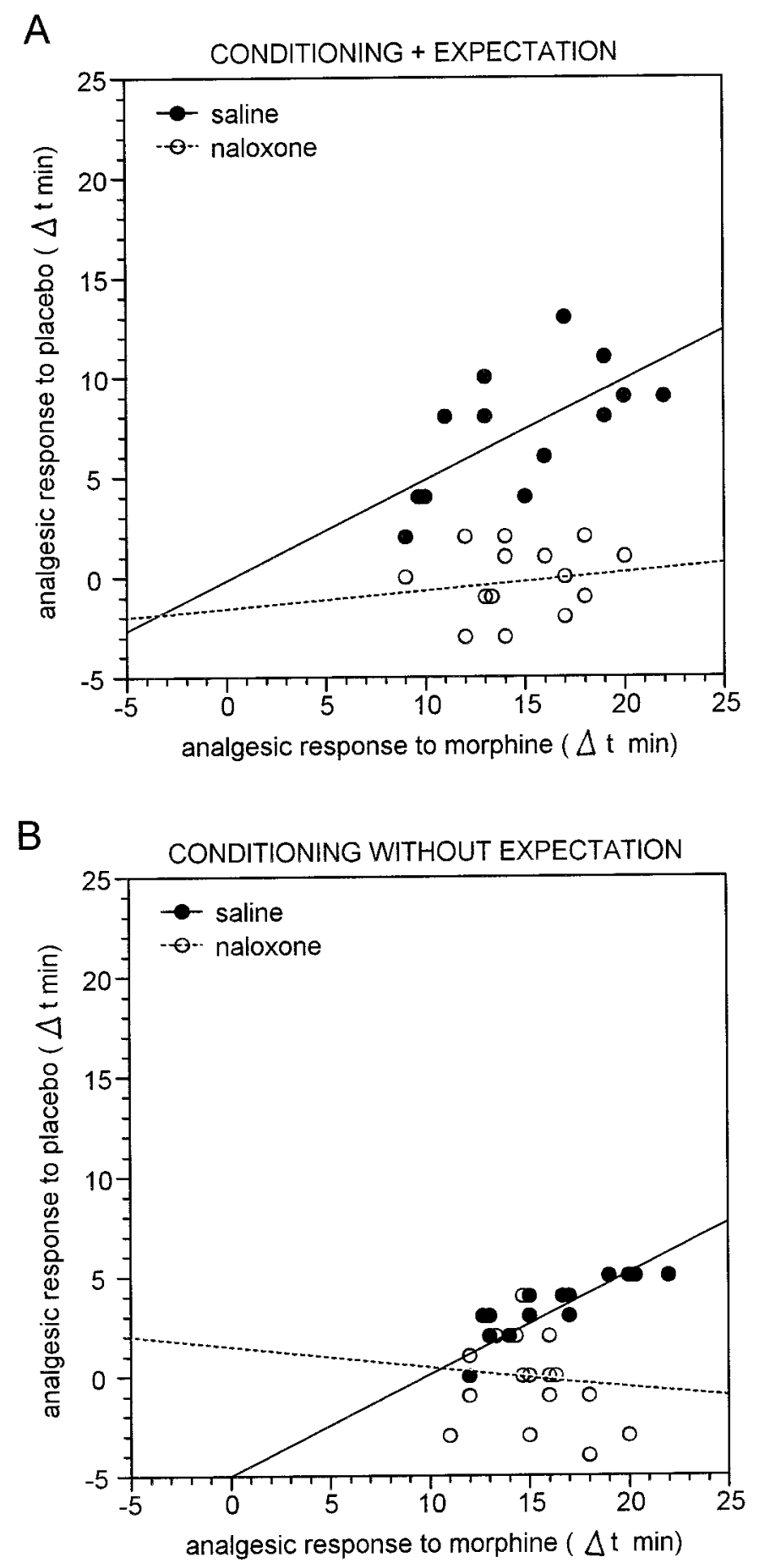

Figure 5. Relationship between the analgesic response to morphine on day 3 and the placebo analgesic response on day 4. Each circle represents the response of a single subject. The responses are expressed as $\Delta t$, that is, the difference of pain tolerance between days 3 and 4 and day 1. $A$, In group 5 (black circles), the larger the morphine response, the larger the placebo response after a saline injection that is believed to be morphine. This correlation is completely disrupted by naloxone in group 6 (white circles). $B$, Same as in $A$ but the saline injection is believed to be an antibiotic (groups 7 and 8).

Therefore, the placebo responses induced by ketorolac conditioning plus expectation were only partially blocked by naloxone, whereas those induced by ketorolac conditioning alone were naloxone-insensitive.

\section{The naloxone-reversible and naloxone-insensitive components of placebo}

Because of the complex experimental design, a brief summary of the statistical analysis previously described is reported below. Expectation alone (group 3) produces a placebo response that can be blocked completely by naloxone (group 4) (Fig. 8A). Conditioning with morphine plus expectation cues (group 5) produce a placebo effect that is larger than morphine conditioning alone (group 7); both can be blocked completely by naloxone (groups 6 and 8) (Fig. 8B). Conditioning with ketorolac plus expectation cues (group 9) produce a placebo effect that has the tendency $\left(F_{(1,29)}=2.92 ; p=0.098\right)$ to be larger than ketorolac conditioning alone (group 11). The former can be blocked by naloxone only partially (group 10), whereas the latter is completely insensitive to naloxone (group 12) (Fig. 8C).

\section{DISCUSSION}

In the present study we have produced different types of placebo response that can be totally blocked, partially blocked, or totally unaffected by naloxone. This indicates that placebo analgesia can be dissected into opioid and nonopioid components, depending on the procedure used to induce the placebo response. These findings were obtained by using a model of experimental pain that has been shown to be sensitive to morphine (Smith et al., 1966, 1972) and to produce well measurable placebo responses (Grevert et al., 1983; Benedetti, 1996). Most important, this type of experimental ischemic arm pain was found to be unaffected by naloxone (Grevert and Goldstein, 1977, 1978; Benedetti, 1996), a necessary condition when naloxone is used to study placebo analgesia. Therefore, the present results are in accordance with previous investigations, confirming that naloxone per se does not influence ischemic arm pain (Fig. $3 A$ ). In addition, we also produced an increase of pain tolerance by means of ketorolac, an NSAID with powerful analgesic activity and no opioid action. It should be pointed out that NSAIDs are known to act at peripheral sites during inflammation by inhibiting the cyclo-oxygenase enzyme necessary for the conversion of arachidonic acid into prostaglandins (Levine and Taiwo, 1994). However, recently it was shown that NSAIDs have a central site of action at the spinal level (Malmberg and Yaksh, 1992). Thus, inflammation is not a necessary condition for the analgesic action of NSAIDs, and the findings of the present study show that ketorolac is a powerful analgesic in experimental ischemic arm pain (Fig. 6).

In a previous study (Benedetti, 1996), we observed that the tourniquet technique induces an increase of pain over time that is variable among different subjects. To reduce this variability, we inflated the sphygmomanometer cuff up to $300 \mathrm{mmHg}$, maintained the Esmarch bandage around the forearm throughout the test, and used a hand exerciser with a force of $7.2 \mathrm{~kg}$. These modifications, compared with the study by Benedetti (1996), produced a quick increase of pain, such that pain tolerances were reduced and variability decreased. In addition, drugs were administered $10 \mathrm{~min}$ before cuff inflation, so that a long time interval was allowed for the drug to produce its effects ( $\sim 25 \mathrm{~min} ; 11 \mathrm{~min}$ before the last squeeze plus $\sim 14 \mathrm{~min}$ of pain tolerance). Therefore, by reducing both pain tolerance and variability, and by maintaining constant the time interval for drug peak effects, we could obtain homogenous populations of subjects. In addition, the use of tolerance as a measure of pain needs some considerations. In fact, tolerance is a complex variable in which the motivationalaffective dimension of pain appears to be more important than the sensory dimension (Price, 1988). We measured pain tolerance 

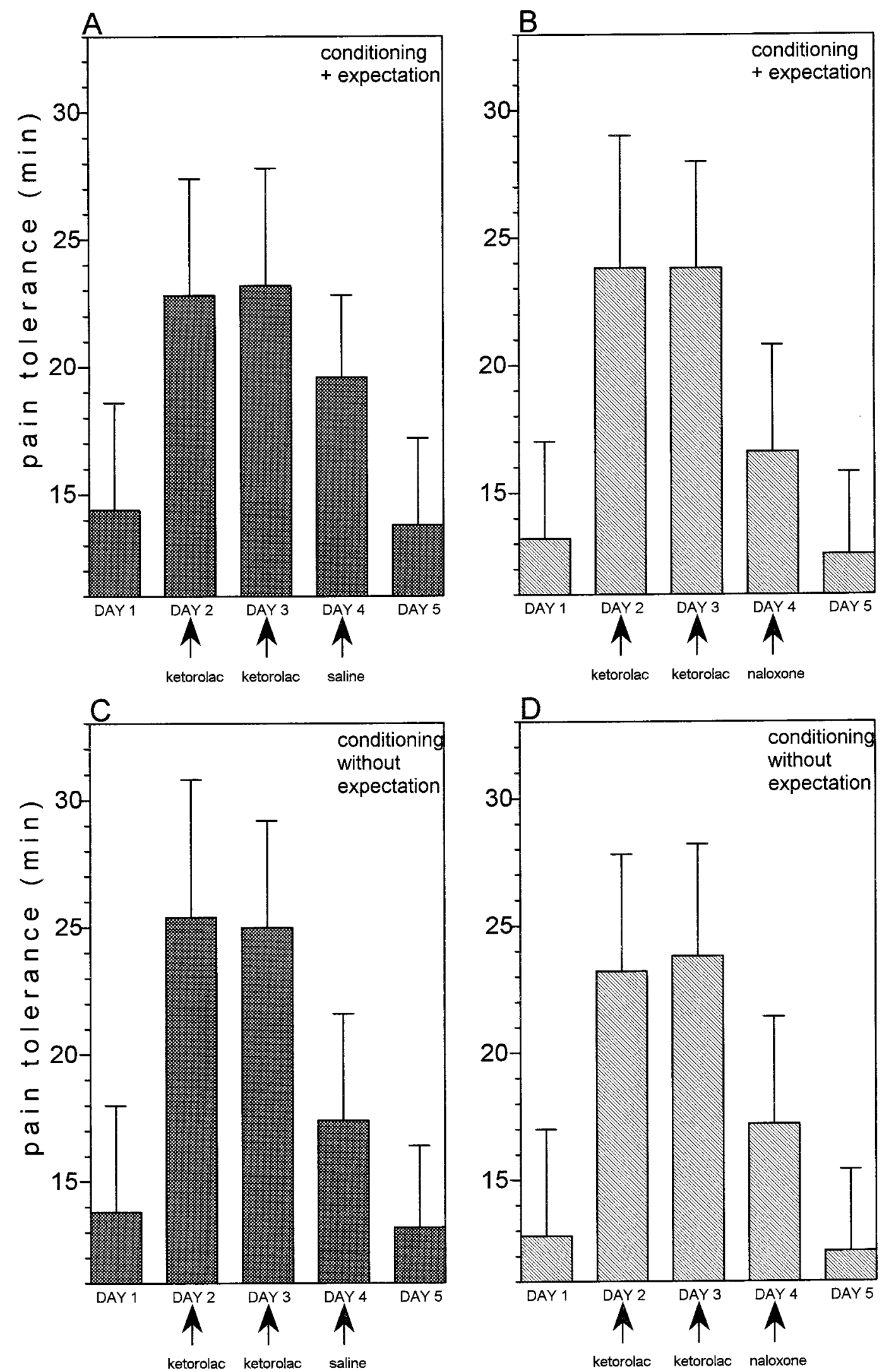

Figure 6. A, After ketorolac conditioning on days 2 and 3, an open injection of saline that is believed to be ketorolac (group 9) mimicks the ketorolac analgesic response. $B$, An open injection of naloxone, which is believed to be ketorolac (group 10), blocks the ketorolac-mimicking effect only partially. $C$, After ketorolac conditioning for 2 consecutive days, an open injection of saline that is believed to be an antibiotic (group 11), mimicks the ketorolac response. $D$, An open injection of naloxone, which is believed to be an antibiotic (group 12), is ineffective in abolishing the ketorolac-mimicking effect. Preconditioning and postconditioning control tests are shown on days 1 and 5 in all cases. 

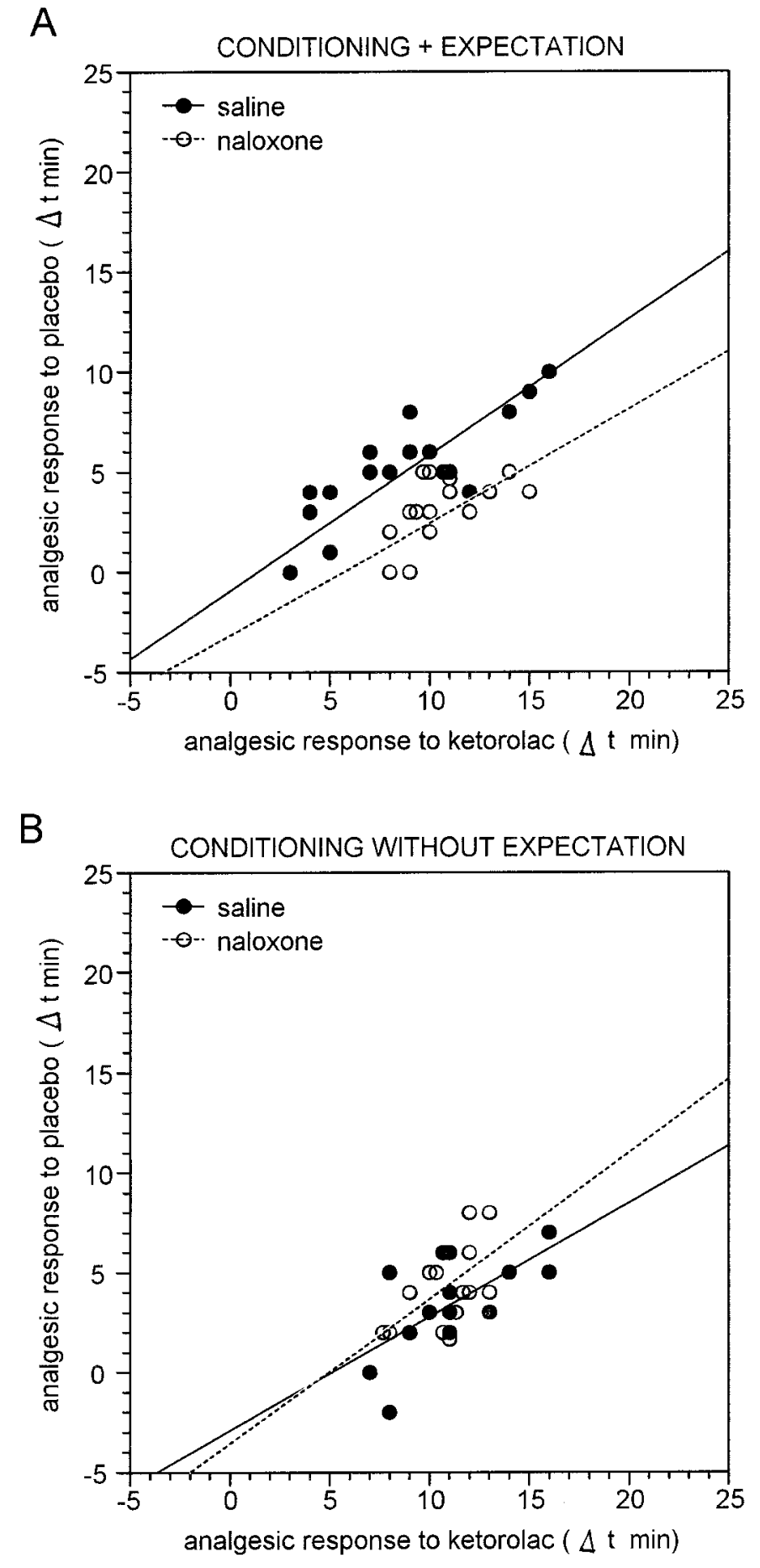

Figure 7. Relationship between the analgesic response to ketorolac on day 3 and the placebo analgesic response on day 4. Each circle represents the response of a single subject. As in Figure 5, the responses are expressed as $\Delta \mathrm{t}$, that is, the difference of pain tolerance between days 3 and 4 and day 1. A, In group 9 (black circles), the larger the ketorolac response, the larger the placebo response after a saline injection that is believed to be ketorolac. This correlation is maintained after naloxone injection in group 10 (white circles). $B$, Same as in $A$ but the saline injection is believed to be an antibiotic (groups 11 and 12). In this case, naloxone is completely ineffective in disrupting the correlation. because it has been shown to be affected by analgesics like morphine (Smith et al., 1966), thus indicating that such a measure of pain can be used to test analgesic drugs. Accordingly, we wanted to see whether placebos produced analgesic-like effects, that is, an increase in tolerance. Even if tolerance measures both the sensory and the motivational-affective component of pain, as carefully stated by Price (1988), this is not against our findings.

One of the main findings emerging from this study is that cognitive factors like expectation appear to trigger endogenous opioid systems in all cases. When we talk of expectation, we refer to verbal expectation. In fact, the subjects believed to receive an analgesic, such that they expected a relief of pain. Although we have not actually measured a change in expectation, the verbal cues (analgesic or antibiotic) are clearly directed in two opposite directions: the first increasing, the second reducing expectation. Unfortunately, we do not know whether in group 3 (expectation) a previous conditioning occurred (Fig. $8 A$ ). In fact, most of the subjects had a previous experience with either opioids or nonopioids (e.g., headache or surgery). Nonetheless, the conditioning experiments with morphine and ketorolac clearly indicate that expectation-induced placebo responses are mediated by endogenous opioids. For example, it is worth emphasizing that ketorolac conditioning alone was naloxone-insensitive, whereas ketorolac conditioning plus expectation was partially naloxone-reversible (Fig. $8 C$ ). This indicates that, by adding expectation cues, an opioid component comes out.

On the other hand, conditioning-induced placebo responses are not mediated by endogenous opioids per se but by specific subsystems, depending on the drug used for conditioning (Fig. 9). If an opioid like morphine is used, conditioning occurs via opioid receptors such that the resulting conditioned placebo response will be naloxone-reversible. Conversely, if conditioning is performed with a nonopioid drug like ketorolac, the resulting placebo response will be naloxone-insensitive. This is probably caused by the involvement of specific mechanisms during conditioning. For instance, the NSAIDs, like ketorolac, act at both peripheral and central sites in the spinal cord (Malmberg and Yaksh, 1992), inhibiting the cyclo-oxygenase enzyme necessary for the conversion of arachidonic acid into prostaglandins. Therefore, conditioning might occur via these nonopioid pathways. We further propose that, if other analgesics (e.g., the $\alpha 2$ adrenergic receptor agonist clonidine or the tricyclic-type antidepressant amitriptyline) are used for conditioning, other mechanisms may result to be involved (e.g., via adrenergic pathways), thus producing a naloxone-insensitive placebo analgesia (Fig. 9).

These findings clarify some previous contrasting studies showing that placebo analgesia is unaffected (Gracely et al., 1983) or reversed (Levine et al., 1978; Grevert et al., 1983; Benedetti, 1996) by naloxone. In fact, if we ignore the strength of the expectation cues and the previous experience (conditioning) with opioids or nonopioids, different subjects with different past experiences can be mistakenly considered to be homogeneous. This issue was first raised by Fields and Levine (1984), who analyzed the different circumstances that might determine whether the placebo response has an opioid component. In particular, Fields and Levine stressed that complex psychological factors, such as instructions, consent form, remuneration, time of placebo administration, method of pain rating, site, and cause of pain may be relevant for the activation of endogenous opioid systems. Thus, it is not surprising that previous studies found placebo effects that respond totally, partially, or do not respond at all to naloxone. If, for example, expectation cues are not adequate, and the subject 

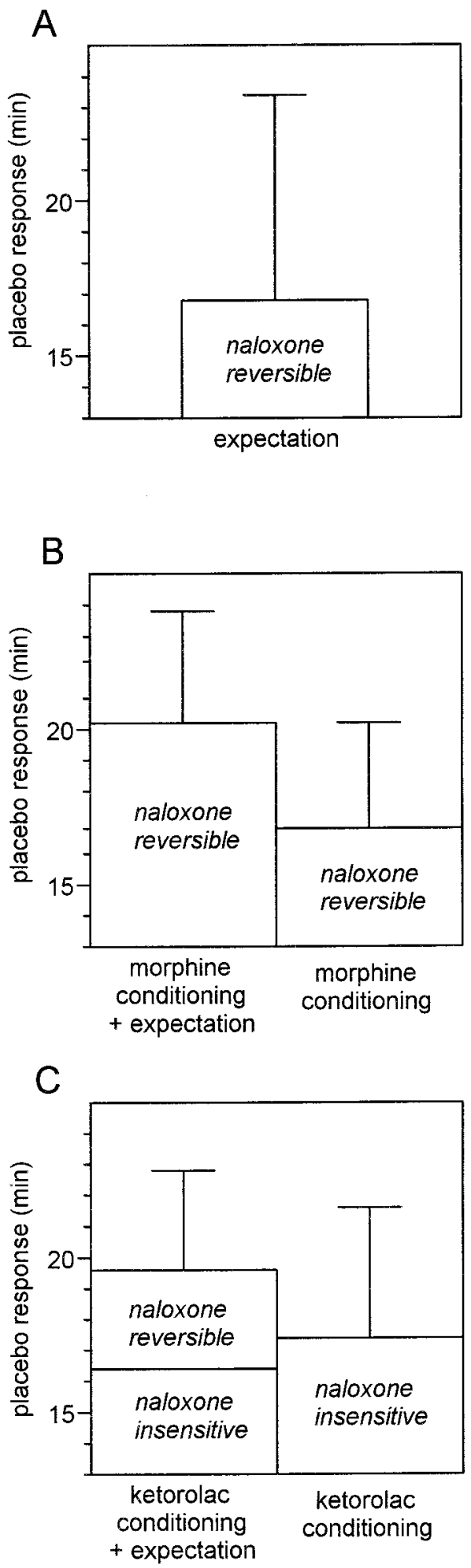

Figure 8. Dissection of placebo analgesia into naloxone-reversible and naloxone-insensitive components. $A$, Expectation-induced placebo analgesia is completely blocked by naloxone. $B$, Morphine conditioning plus expectation produces a placebo response that is totally blocked by naloxone. Morphine conditioning alone induces placebo responses that, similarly, are completely blocked by naloxone. $C$, Nonopioid ketorolac conditioning plus expectation produces a placebo response that is only partially antagonized by naloxone. By contrast, nonopioid ketorolac conditioning alone induces placebo responses that are completely insensitive to naloxone. has been previously conditioned with nonopioid drugs, the placebo response is likely to be naloxone-insensitive. By contrast, if the subject had a previous experience with opioids, and the expectation cues are strong, the placebo response will result to be naloxone-reversible.

It is interesting that placebo responses occurred even without expectation of pain relief. In other words, if the subject was previously conditioned with either morphine or ketorolac, the lack of expectation cues only reduced but did not prevent the occurrence of a placebo effect. These findings are in agreement with those by Voudouris et al. (1990), who showed that conditioned placebo responses can be obtained without expectancy. Thus, previously conditioned subjects experience an analgesic effect even if they do not expect it. Nonetheless, it should be reminded that in a recent work, Montgomery and Kirsch (1997) showed that placebo analgesia can result from conditioning but is mediated by expectation. This is consistent with our findings that, by reducing expectation in conditioned subjects (belief to receive an antibiotic), the placebo effect results to be smaller. However, this small residual effect is likely to represent a sequence effect caused by learning (conditioning), with little or no involvement of expectation. This notion is supported by a recent study (Benedetti et al., 1999), showing that a similar conditioning can be found in placebo respiratory depression, a phenomenon mediated by endogenous opioids and in which expectation cues are not present.

Several studies showed that conditioning plays an important

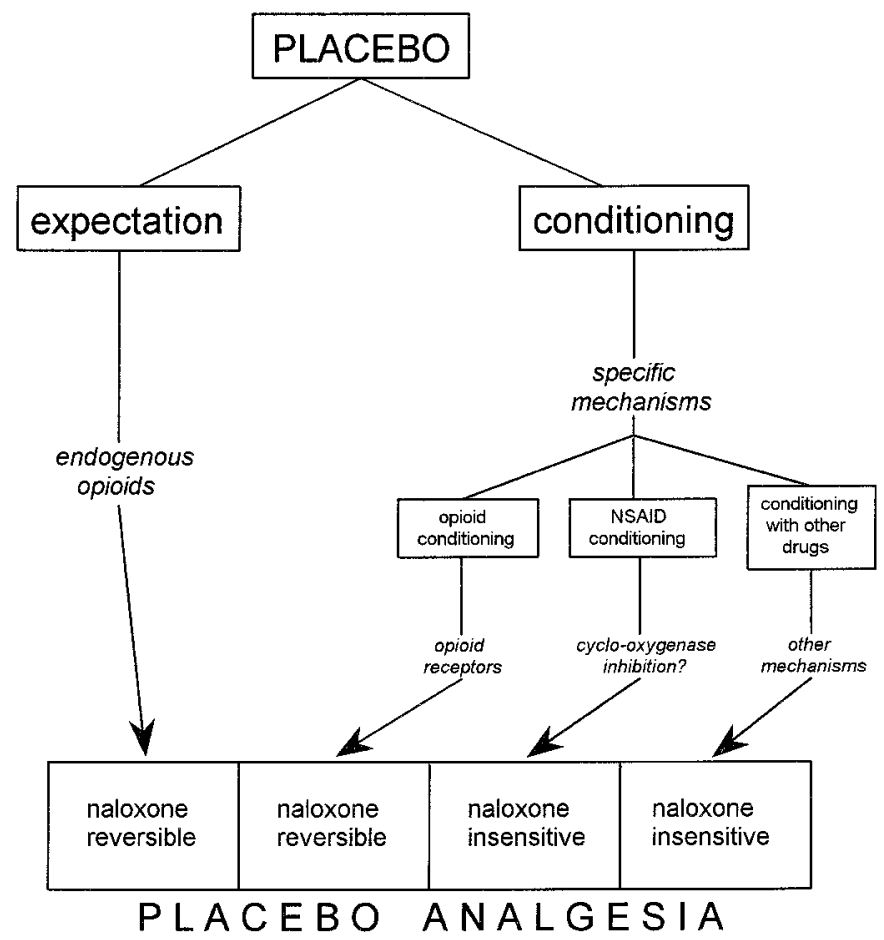

Figure 9. Schematic diagram of the mechanisms activating endogenous opioid systems and nonopioid systems in placebo analgesia. The administration of a placebo can trigger both cognitive (expectation) and conditioning mechanisms. Expectation activates endogenous opioid systems, whereas conditioning is mediated by specific mechanisms. If conditioning is performed with opioids, placebo analgesia is mediated via opioid receptors. However, if conditioning is performed with nonopioid drugs, other mechanisms result to be involved. Therefore, placebo analgesia can result to be either naloxone-reversible or partially naloxone-reversible or, otherwise, naloxone-insensitive, depending on the procedure used to evoke the placebo response. 
role in the placebo response, and this is true for pain, the immune system and, in general, for pharmacotherapy (Gleidman et al., 1957; Herrnstein, 1962; Batterman, 1966; Batterman and Lower, 1968; Laska and Sunshine, 1973; Ader, 1985; Siegel, 1985; Wickramasekera, 1985; Voudouris et al., 1989, 1990; Ader, 1997; Benedetti et al., 1998). Similarly, cognitive and motivational factors, such as expectation and desire of pain relief, appear to play an essential role (Fields and Price, 1997; Price and Fields, 1997). The findings of the present study and the experimental approach by itself show that cognition and conditioning can be balanced in different ways during a placebo procedure. This balance is crucial for the activation of opioid systems or other specific subsystems and has at least three important implications. First, a complex cognitive function, like expectation of pain relief, is capable to interact with neurochemical systems and to produce a specific analgesic effect. Second, the placebo response depends on past experience, being mediated by specific subsystems that are likely to be activated during learning. Third, the understanding of the intricate mechanisms linking mental activity and pain will help in planning new therapeutic strategies.

\section{REFERENCES}

Ader R (1985) Conditioned immunopharmacological effects in animals: implications for a conditioning model of pharmacotherapy. In: Placebo: theory, research, and mechanisms (White L, Tursky B, Schwartz GE, eds), pp 306-323. New York: Guilford.

Ader R (1997) The role of conditioning in pharmacotherapy. In: The placebo effect: an interdisciplinary exploration (Harrington A, ed), pp 138-165. Cambridge, MA: Harvard UP.

Amanzio M, Pollo A, Benedetti F (1998) Endogenous opioids mediate both placebo analgesia and placebo respiratory depression. Soc Neurosci Abstr 495.11.

Batterman RC (1966) Persistence of responsiveness with placebo therapy following an effective drug trial. J New Drugs 6:137-141.

Batterman RC, Lower WR (1968) Placebo responsiveness: influence of previous therapy. Curr Ther Res 10:136-143.

Benedetti F (1996) The opposite effects of the opiate antagonist naloxone and the cholecystokinin antagonist proglumide on placebo analgesia. Pain 64:535-543.

Benedetti F, Amanzio M (1997) The neurobiology of placebo analgesia: from endogenous opioids to cholecystokinin. Prog Neurobiol 52:109-125.

Benedetti F, Amanzio M, Maggi G (1995) Potentiation of placebo analgesia by proglumide. Lancet 346:1231.

Benedetti F, Amanzio M, Baldi S, Casadio C, Cavallo A, Mancuso M, Ruffini E, Oliaro A, Maggi G (1998) The specific effects of prior opioid exposure on placebo analgesia and placebo respiratory depression. Pain 75:313-319.

Benedetti F, Amanzio M, Baldi S, Casadio C, Maggi G (1999) Inducing placebo respiratory depressant responses in humans via opioid receptors. Eur J Neurosci, in press.

Evans FJ (1985) Expectancy, therapeutic instructions, and the placebo response. In: Placebo: theory, research, and mechanisms (White L, Tursky B, Schwartz GE, eds), pp 215-228. New York: Guilford.

Fields HL, Levine JD (1984) Placebo analgesia: a role for endorphins? Trends Neurosci 7:271-273.

Fields HL, Price DD (1997) Toward a neurobiology of placebo analge- sia. In: The placebo effect: an interdisciplinary exploration (Harrington A, ed), pp 93-116. Cambridge, MA: Harvard UP.

Gleidman LH, Grantt WH, Teitelbaum HA (1957) Some implications of conditional reflex studies for placebo research. Am J Psychiatry 113:1103-1107.

Gracely RH, Dubner R, Wolskee PJ, Deeter WR (1983) Placebo and naloxone can alter postsurgical pain by separate mechanisms. Nature 306:264-265.

Grevert P, Goldstein A (1977) Effects of naloxone on experimentally induced ischemic pain and on mood in human subjects. Proc Natl Acad Sci USA 74:1291-1294.

Grevert P, Goldstein A (1978) Endorphins: naloxone fails to alter experimental pain or mood in humans. Science 199:1093-1095.

Grevert P, Albert LH, Goldstein A (1983) Partial antagonism of placebo analgesia by naloxone. Pain 16:129-143.

Herrnstein RJ (1962) Placebo effect in the rat. Science 138:677-678.

Laska E, Sunshine A (1973) Anticipation of analgesia: a placebo effect. Headache 13:1-11.

Levine JD, Gordon NC (1984) Influence of the method of drug administration on analgesic response. Nature 312:755-756.

Levine JD, Taiwo Y (1994) Inflammatory pain. In: Textbook of pain (Wall PD, Melzack R, eds), pp 45-56. Edinburgh: Churchill Livingstone.

Levine JD, Gordon NC, Fields HL (1978) The mechanism of placebo analgesia. Lancet 2:654-657.

Malmberg AB, Yaksh TL (1992) Hyperalgesia mediated by spinal glutamate or substance $\mathrm{P}$ receptor blocked by spinal cyclo-oxygenase inhibition. Science 257:1277-1280.

Montgomery GH, Kirsch I (1997) Classical conditioning and the placebo effect. Pain 72:107-113.

Price DD (1988) Psychological and neural mechanisms of pain. New York: Raven.

Price DD, Fields HL (1997) The contribution of desire and expectation to placebo analgesia: implications for new research strategies. In: The placebo effect: an interdisciplinary exploration (Harrington A, ed), pp 117-137. Cambridge, MA: Harvard UP.

Siegel S (1985) Drug anticipatory responses in animals. In: Placebo: theory, research, and mechanisms (White L, Tursky B, Schwartz GE, eds), pp 288-305. New York: Guilford.

Smith GM, Egbert LD, Markowitz RA, Mosteller F, Beecher HK (1966) An experimental pain method sensitive to morphine in man: the submaximum effort tourniquet technique. J Pharmacol Exp Ther 154:324-332.

Smith GM, Lowenstein E, Hubbard JH, Beecher HK (1972) Experimental pain produced by the submaximum effort tourniquet technique: further evidence of validity. J Pharmacol Exp Ther 163:468-474.

ter Riet G, de Craen AJM, de Boer A, Kessels AGH (1998) Is placebo analgesia mediated by endogenous opioids? A systematic review. Pain 76:273-275.

Voudouris NJ, Peck CL, Coleman G (1989) Conditioned response models of placebo phenomena: further support. Pain 38:109-116.

Voudouris NJ, Peck CL, Coleman G (1990) The role of conditioning and verbal expectancy in the placebo response. Pain 43:121-128.

Wall PD (1992) The placebo effect: an unpopular topic. Pain 51:1-3.

Wall PD (1993) Pain and the placebo response. In: Ciba foundation symposium 174, experimental and theoretical studies of consciousness, pp 187-216. New York: Wiley.

Wickramasekera I (1985) A conditioned response model of the placebo effect: predictions from the model. In: Placebo: theory, research, and mechanisms (White L, Tursky B, Schwartz GE, eds), pp 255-287. New York: Guilford. 